EPJ Web of Conferences 104, 01006 (2015)

DOI: $10.1051 /$ epjconf/201510401006

(C) Owned by the authors, published by EDP Sciences, 2015

\title{
Inelastic neutron scattering applied to materials for energy
}

\author{
Andrea Piovano ${ }^{\mathrm{a}}$ \\ Institut Laue-Langevin (ILL), BP. 156 X, 38042 Grenoble Cedex, France
}

\begin{abstract}
Inelastic neutron scattering measures the dynamical processes in materials, i.e. the spontaneous fluctuations that experiences samples when interacting with neutrons. An inelastic neutronspectrum is obtained from measurements of both energy and the momentum transfer between the neutron and the sample. This short dissertation explains fundamental concepts of the inelastic neutron scattering process, describes instrumentation, and gives a couple of materials applications were inelastic neutron scattering has been proven to be useful like ionic conductors and thermoelectric materials.
\end{abstract}

\section{Introduction}

Creating a environmentally and economically sustainable energy-system is one of the largest challenges that scientific and engineering communities have to face. Alternative energy-technologies and new materials have risen as a result of the needs to move away from fossil fuels and reduce pollutant emissions. Neutron based techniques of analysis play a role in almost all aspects of sustainable-energy materials research, and the chapters of this book will focus on studies made by using inelastic neutron scattering and using examples and case studies to illustrate research approaches, methods, and outcomes.

The research on renewable materials of relevance is one of the most rapidly-growing research areas in materials science. The primary challenge in this research is the development of materials that are viable in competition with existing energy-technologies, responding to application requirements such as efficiency, durability, and cost. Understanding the fundamental properties of materials and their functionality at the atomic and molecular level is crucial in addressing the global challenge of cleaner sources of renewable energy.

A key requirement for the prediction of thermodynamic properties is an accurate description of the phonon density of states. Inelastic neutron scattering (INS) measurements using single-crystal and powder samples provide complete measurements of vibrational properties, including the phonon dispersion relation and phonon density of states. Studies of these phonon properties are of immense interest as they provide valuable quantitative information concerning thermodynamic properties and phase instabilities. Neutron scattering experiments have played a key role in validating theoretical models, which in turn provide microscopic insights to a variety of novel phenomena.

Lattice dynamics calculations in turn have played a key role in the planning, analysis, and interpretation of INS experiments in these structurally complex solids. Quantum mechanical first

\footnotetext{
${ }^{a}$ Corresponding author: piovano@ill.fr
}

This is an Open Access article distributed under the terms of the Creative Commons Attribution License 4.0, which permits unrestricted use, distribution, and reproduction in any medium, provided the original work is properly cited. 


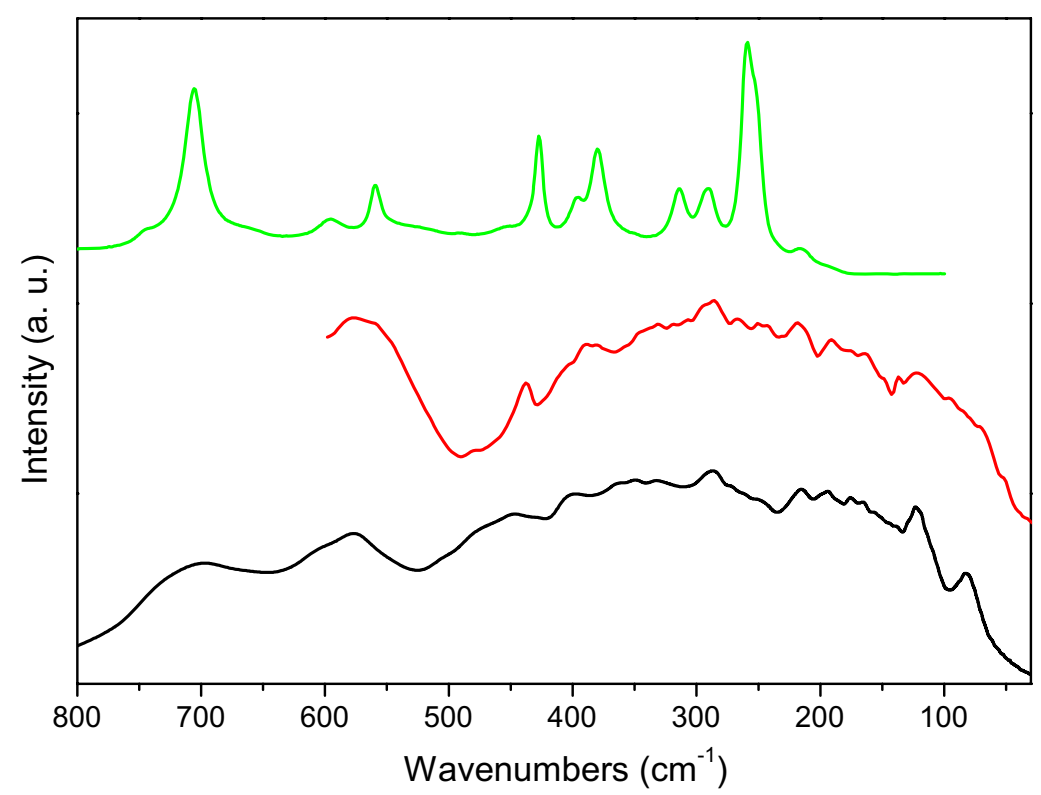

Figure 1. $\mathrm{CaFeO}_{25}$ polycrystalline sample measured with Raman (green curve), with ATR infrared (red curve) and by INS (black curve).

principles calculations have been very effective in elucidating microscopic details about the vibrational spectra, elastic behaviour, and thermodynamic properties of several systems.

The most common methods for studying molecular vibrations are the well established optical techniques of infrared and Raman spectroscopy. It is through a direct comparison with these techniques that the information that inelastic neutron scattering can give are highlighted, as shown in Fig. 1 for a polycrystalline sample of $\mathrm{CaFeO}_{2.5}$. Measured INS intensities are directly related to the atomic displacements of the scattering atom. Any complications arising from the electro-optic parameters are avoided. Indeed the band positions and intensities of most molecular systems can be accurately calculated using modern ab initio computational methods.

All vibrations are active in inelastic neutron scattering and measurable. Moreover the scattered the neutron transfers also momentum to the atom and so inelastic neutron measurements are not limited to observation at the Brillouin zone centre as are photon techniques. Neutrons penetrate deeply, of the order of millimeters, into typical samples and results are thus naturally weighted to the measurement of bulk properties. Inelastic neutron spectrometers cover a wide vibrational range and more importantly the lower energy range (below $50 \mathrm{meV}$ ) is readily accessible, a region that is experimentally more difficult for infrared and Raman spectroscopies.

This chapter is intended to show the fundamental aspect of inelastic neutron scattering technique as probe of lattice dynamic behavior of materials, with focus on systems with an importance in the energy field.

The theory of neutron scattering is well established and interested reader can refer to seminal book or more recent manuscript for a detailed development of it [1-3]. Here we will limit to show the most important relation that make inelastic neutron spectroscopy a so important technique to understand dynamics of materials as it directly probe the spontaneous fluctuations present in them.

Several instrumentations are available for the analysis of the dynamics of systems using neutron as probe. Here the two most important of these spectrometers, the triple axis one and the time of flight one, 
will be presented prior to show a couple of examples in which they have been helpful in understanding the properties of two materials for energy, namely a oxygen ion conductor and a thermoelectric material.

\section{Inelastic neutron scattering}

\subsection{General remarks}

The goal of the study of excitations in solids is to understand on a microscopic level their properties. Then the important step is to find a way to link the response of a system to an external perturbation to the thermodynamic properties at equilibrium. The mathematical basis to do this step is the theory of linear response. We cannot develop it here, but its importance is in the fact that if a perturbation $\mathrm{F}$ is small, the changes to a generic variable of the system A will be linear in principle, i.e. the response will be proportional to the amplitude and to the duration of the perturbation.

The Fourier transform of $\mathrm{A}(\mathrm{t})$ and $\mathrm{F}(\mathrm{t})$ in the of frequency domain is related by the so called generalized susceptibility which is the Laplace transform of the response function $\phi(t)$ so that:

$$
A(\omega)=\chi[\omega] F(\omega) .
$$

The response to a periodic perturbation is in turn periodic with the same frequency. The generalized susceptibility is a complex function

$$
\chi[\omega]=\chi^{\prime}[\omega]+i \chi^{\prime \prime}[\omega]
$$

where the real part describe the response in phase with the perturbation (reactive part), while the imaginary part is connected with the response out of phase (dissipative part). The fluctuation-dissipation theorem connect the susceptibility to the auto-correlation function $S(\omega)$ by:

$$
\mathrm{S}(\omega)=(\mathrm{n}(\omega)+1) \chi^{\prime \prime}[\omega],
$$

through the thermal occupation factor

$$
\mathrm{n}(\omega)=\frac{1}{\exp \left(\frac{\mathrm{h} \omega}{\mathrm{K}_{\mathrm{B}} \mathrm{T}}\right)-1} .
$$

We will see that $S(\omega)$, with the name of scattering function, plays a master role in the theory of inelastic scattering.

\subsection{Scattering cross section and scattering length}

If we consider a mono-energetic beam of neutrons with wave vector $\mathbf{k}_{\mathrm{i}}$ and flux $\phi\left(\mathbf{k}_{\mathrm{i}}\right)$ incident on a sample, the rate at which they are scattered by it is given by the product of the incoming flux with the scattering cross section $\sigma$. In a neutron scattering experiment, we observe neutron scattered into a specific solid angle $\mathrm{d} \Omega_{\mathrm{f}}$, in the direction of the wave vector $\mathbf{k}_{\mathrm{f}}$ with a final energy between $\mathrm{E}_{\mathrm{f}}$ and $\mathrm{E}_{\mathrm{f}}+$ $\mathrm{dE}_{\mathrm{f}}$. This rate is given by the product of $\phi\left(\mathbf{k}_{\mathrm{i}}\right)$ with the double differential cross section $\mathrm{d}^{2} \sigma / \mathrm{d} \Omega_{\mathrm{f}} \mathrm{dE}_{\mathrm{f}}$.

As a probe for condensed matter, the neutron acts as a weak perturbation of the scattering system and so it can cause a transition in between quantum states, but not modify the nature of the state. As a consequence the differential cross section can be obtained from the Fermi's Golden Rule:

$$
\left.\frac{d^{2} \sigma}{d \Omega_{f} d E_{f}}\right|_{\mathrm{l}_{\mathrm{i}} \rightarrow \mathrm{l}_{\mathrm{f}}}=\frac{k_{f}}{k_{i}}\left(\frac{m_{n}}{2 \pi \hbar^{2}}\right)^{2}\left|\left\langle\boldsymbol{k}_{f} l_{f}|V| \boldsymbol{k}_{i} l_{i}\right\rangle\right|^{2} \delta\left(\hbar \omega+E_{i}-E_{f}\right),
$$

where $l_{i}$ an $l_{f}$ the initial and final quantum states of the sample and $V$ is the interaction operator for the neutrons with the sample, which represents the nuclear potential. Due to the very short time of interaction the potential is essentially a delta function and it can be expressed in the terms of Fermi 
pseudo-potential

$$
V(\boldsymbol{Q})=\frac{2 \pi \hbar^{2}}{m_{n}} b
$$

where $b$ is the nuclear scattering length.

This cross section could be split in the coherent and incoherent parts

$$
\frac{d^{2} \sigma}{d \Omega_{f} d E_{f}}=\left.\frac{d^{2} \sigma}{d \Omega_{f} d E_{f}}\right|_{\text {coh }}+\left.\frac{d^{2} \sigma}{d \Omega_{f} d E_{f}}\right|_{\text {inc }},
$$

as the coherent part give information on the cooperative effects among different atoms, while the incoherent on the individual particle motion.

It is worth explaining here the difference in between the coherent and incoherent cross sections and how they relate respect to the scattering length. Indeed, even a monoatomic sample may contain nuclei with different scattering length, as the different isotopes of the same nuclei have different $b$. Consider now the scattering length $b_{\mathrm{n}}$ of a $n$ distinct isotope occurring with a frequency $\mathrm{f}$. If there are no correlations in between the nuclear position and the scattering length, the scattering will depend only on the average (or coherent) scattering length

$$
\bar{b}=\sum_{n} f_{n} b_{n} .
$$

The average coherent cross section per atom becomes

$$
\sigma_{c o h}=4 \pi(\bar{b})^{2} .
$$

The random fluctuations in the scattering length from atom to atom will not contribute to the collective scattering, but only to the incoherent scattering

$$
\sigma_{i n c}=4 \pi\left(\overline{b^{2}}-\bar{b}^{2}\right)
$$

with the effective incoherent scattering length

$$
b_{i n c}=\sqrt{\overline{b^{2}}-\bar{b}^{2}} .
$$

\subsection{Scattering function}

We have already mentioned that the neutron matter interaction is weak. This means that we can evaluate the interaction matrix elements using the Born approximation and treating the incoming and outgoing neutrons as plane waves. Furthermore in a scattering experiment, where many transitions of the type described in Eq. (2.5), it is necessary to average over initial states (each one weighted by event probability) and sum over all final states. After some mathematical manipulation that can be found in textbooks the differential cross section can be expressed as

$$
\frac{d^{2} \sigma}{d \Omega_{f} d E_{f}}=\frac{k_{f}}{k_{i}} S(\boldsymbol{Q}, \omega)
$$

with

$$
S(\boldsymbol{Q}, \omega)=\frac{1}{2 \pi \hbar} \sum_{m, m^{\prime}=1}^{N} \overline{b_{m^{\prime}} b_{m}} \int_{-\infty}^{\infty}\left\langle e^{-i \boldsymbol{Q} \cdot \boldsymbol{r}_{m^{\prime}}(0)} e^{i \boldsymbol{Q} \cdot \boldsymbol{r}_{m}(t)}\right\rangle e^{-i \omega t} d t .
$$

In this form of the scattering function, $N$ is the total number of nuclei, $t$ is the time and the angular brackets denote the average over initial states, and $\boldsymbol{r}_{m}$ are the coordinates of the scattering centres. As it can be seen, the scattering function depends only on the momentum and energy transferred from the 


\section{JDN 21}

neutron to the sample and not on the absolute values of $\boldsymbol{k}_{\mathrm{i}}$ and $\boldsymbol{k}_{\mathrm{f}}$. It contains information on both the position and the motion of the atoms in the sample. The goal of most neutron scattering experiments is to measure the $\mathbf{S}(\mathbf{Q}, \omega)$ and then extract from it the microscopic properties of the system.

On the assumption that no correlation exist among the $b$ values of different nuclei

$$
\begin{array}{ll}
\overline{b_{m^{\prime}} b_{m}}=(\bar{b})^{2}, \quad & m^{\prime} \neq m, \\
\overline{b_{m^{\prime}} b_{m}}=\overline{b^{2}}, & m^{\prime}=m,
\end{array}
$$

We can separate the scattering function in its coherent and incoherent parts as:

$$
\begin{gathered}
S(\boldsymbol{Q}, \omega)_{c o h}=\frac{1}{2 \pi \hbar}(\bar{b})^{2} \sum_{m, m^{\prime}=1}^{N} \int_{-\infty}^{\infty}\left\langle e^{-i \boldsymbol{Q} \cdot \boldsymbol{r}_{m^{\prime}}(0)} e^{i \boldsymbol{Q} \cdot \boldsymbol{r}_{m}(t)}\right\rangle e^{-i \omega t} d t, \\
S(\boldsymbol{Q}, \omega)_{i n c}=\frac{1}{2 \pi \hbar}\left(\overline{b^{2}}-(\bar{b})^{2}\right) \sum_{m=1}^{N} \int_{-\infty}^{\infty}\left\langle e^{-i \boldsymbol{Q} \cdot \boldsymbol{r}_{m}(0)} e^{i \boldsymbol{Q} \cdot \boldsymbol{r}_{m}(t)}\right\rangle e^{-i \omega t} d t .
\end{gathered}
$$

We can more clearly see from these equations that the coherent scattering depends on correlations between the positions of all nuclei (both different or the same) at different times and so gives interference effect. The incoherent scattering depends only on the correlation between the positions of the same nucleus at different times and as consequence does not produce interference effects.

The physical basis for this, resides the different scattering lengths associated with the different nuclei that an actual scattering systems has. The coherent part is the scattering that the system would give if all the scattering length were equal to $\bar{b}$. The incoherent part arises from the random distribution of the deviations of the scattering length of a real system from the mean value.

The inelastic neutron scattering is a method for the microscopic investigation of reciprocal space and energy exchange $(\mathbf{Q}, \omega)$ of a system, with $\mathbf{Q}$ being the wavevector and $\hbar \omega$ the energy. During an experiment what it is measured is the number of neutrons that have been scattered in a certain direction with a certain energy exchange. These information give access to the scattering function of a system $S(\mathbf{Q}, \omega)$. This function is linked to the imaginary part of the dynamical susceptibility by the over mentioned fluctuation-dissipation theorem (see Eq. (2.3)):

$$
S(\mathbf{Q}, \omega)=\frac{\chi^{\prime \prime}(\mathbf{Q}, \omega)}{1-\mathrm{e}^{-\frac{h \omega}{\mathrm{K}_{\mathrm{B}} \mathrm{T}}}} .
$$

The formula applies to both positive and negative values of w: positive w corresponds to a transfer of energy from the neutron to the sample, while negative $w$ to energy transfer from the sample to the neutron.

Once subtracted the elastic contributions, the scattering function include all the fluctuations in the sample in function of momentum and energy. An important property of $\mathbf{S}(\mathbf{Q}, \omega)$ is the principle of detailed balance

$$
\mathrm{S}(-\mathbf{Q},-\omega)=\mathrm{e}^{-\frac{\hbar \omega}{\mathrm{K}_{\mathrm{B}} \mathrm{T}} \mathrm{S}} \mathrm{S}(\mathbf{Q}, \omega)
$$

where $K_{B}$ is the Boltzmann constant and points out that the transition probability in the sample depends on the statistical weight for the initial state, which will be lower for the annihilation of an excitation that for the creation of an excitation. Due to the relation $S(-\mathbf{Q}, \omega)=\mathbf{S}(\mathbf{Q}, \omega)$, the detailed balance principle requires $\chi^{\prime \prime}(\mathbf{Q}, \omega)$ to be an odd function, like the thermal factor, so that $\mathbf{S}(\mathbf{Q}, \omega)$ remains always positive. 


\subsection{Nuclear scattering by crystals}

The scattering function in Eq. (2.13) does not depend on the intrinsic properties of neutron. If we neglect the spin, the properties of neutron can be reported to the own wavevector $\mathbf{k}$ only. The $S(\mathbf{Q}, \omega)$ still include the interaction potential with that depend on the scattering length $b_{\mathrm{m}}$.

Now we can look how we can develop the scattering function when neutrons scatter an ordered crystal. Consider a crystal with unit cell sides defined by $\boldsymbol{a}_{1}, \boldsymbol{a}_{2}, \boldsymbol{a}_{3}$ and with reciprocal lattice unit-cell vectors $\boldsymbol{a}_{1}^{*}, \boldsymbol{a}_{2}^{*}, \boldsymbol{a}_{3}^{*}$. Due to thermal motion the nucleus is displaced from its equilibrium position $m$ so that its instantaneous position is

$$
\boldsymbol{R}_{m}(t)=\boldsymbol{m}+\boldsymbol{u}_{m}(t),
$$

where $\boldsymbol{u}_{m}(t)$ is the Heisemberg operator for the displacement. Assuming that the interatomic forces in the crystal are harmonic, i.e. that the forces are linear functions of the displacements, $\boldsymbol{u}_{m}(t)$ can be expressed as a sum of a set of normal modes

$$
\boldsymbol{Q} \cdot \boldsymbol{u}_{m}(t)=\left(\frac{\hbar}{2 M N}\right)^{1 / 2} \sum_{s} \frac{\boldsymbol{q} \cdot \boldsymbol{e}_{s}}{\sqrt{\omega_{s}}}\left[a_{s} e^{i\left(\boldsymbol{Q} \cdot \boldsymbol{m}-\omega_{s} t\right)}+a_{s}^{+} e^{-i\left(\boldsymbol{Q} \cdot \boldsymbol{m}-\boldsymbol{\omega}_{s} t\right)}\right],
$$

where $\boldsymbol{q}$ is the wavevector of the mode, $\omega_{\mathrm{s}}$ is the angular frequency of mode $s$, and $\mathbf{e}_{\mathrm{s}}$ is its polarization vector. The sum over s is over the $N$ values of $q$ in the first Brillouin zone. $M$ is the mass of an atom, assumed to be the same for all the atoms. $a_{s}(t)$ is the annihilation Heisenberg operator for the mode $s$, and $a_{s}^{+}(t)$, its Hermitian conjugate, the creation operator. In this way the scattering function (2.13) can be rewritten as

$$
S(\boldsymbol{Q}, \omega)_{c o h}=\frac{N}{2 \pi \hbar}(\bar{b})^{2} \sum_{m} e^{-i(\boldsymbol{Q} \cdot \boldsymbol{m})} \int_{\infty}^{\infty}\left\langle e^{U} e^{V}\right\rangle e^{-i \omega t} d t
$$

with

$$
\begin{gathered}
U=-i \boldsymbol{Q} \cdot \boldsymbol{u}_{m}(0)=-i \sum_{s} g_{s} a_{s}+g_{s} a_{s}^{+}, \\
V=-i \boldsymbol{Q} \cdot \boldsymbol{u}_{m}(t)=i \sum_{s} h_{s} a_{s}+h_{s}^{*} a_{s}^{+},
\end{gathered}
$$

and

$$
\begin{aligned}
& g_{s}=\left(\frac{\hbar}{2 M N}\right)^{1 / 2} \frac{\boldsymbol{q} \cdot \boldsymbol{e}_{s}}{\sqrt{\omega_{s}}} \\
& h_{s}=\left(\frac{\hbar}{2 M N}\right)^{1 / 2} \frac{\boldsymbol{q} \cdot \boldsymbol{e}_{s}}{\sqrt{\omega_{s}}} e^{i\left(\boldsymbol{Q} \cdot \boldsymbol{m}-\omega_{s} t\right)} .
\end{aligned}
$$

It is possible to demonstrate that the term $\langle\exp U \exp V\rangle$ can be developed in such a way to obtain

$$
\left\langle e^{U} e^{V}\right\rangle=e^{\left\langle U^{2}\right\rangle} e^{\langle U V\rangle},
$$

so that the coherent scattering function take the form

$$
S(\boldsymbol{Q}, \omega)_{c o h}=\frac{N}{2 \pi \hbar}(\bar{b})^{2} e^{\left\langle U^{2}\right\rangle} \sum_{m} e^{-i(\boldsymbol{Q} \cdot \boldsymbol{m})} \int_{-\infty}^{\infty} e^{\langle U V\rangle} e^{-i \omega t} d t .
$$

A crystal of $\mathrm{N}$ atoms has $3 \mathrm{~N}$ normal modes. The initial state of the crystal is given by specifying the quantum numbers of the $3 \mathrm{~N}$ oscillators corresponding to the normal modes. In a scattering process, the state of the crystal changes giving another set of quantum numbers. The scattering process may thus be classified according to the changes in the quantum numbers. If we expand the term $\exp (U V)$ in $(2.25)$ 
we obtain

$$
e^{\langle U V\rangle}=1+\langle U V\rangle+\frac{1}{2 !}\langle U V\rangle^{2}+\ldots+\frac{1}{n !}\langle U V\rangle^{n} .
$$

The $\mathrm{n}$-th term gives the cross-section for all $n$-phonon processes and the process is called phonon expansion. Thus the first term, 1, gives the elastic cross-section. The next term (UV) gives the crosssection for all one-phonon processes, the term $1 / 2 !\langle U V\rangle^{2}$ gives the cross-section for all two-phonon processes and so on. The expression for the coherent one-phonon cross-section become

$$
\begin{aligned}
\left(\frac{d^{2} \sigma}{d \Omega_{f} d E_{f}}\right)_{c o h 1 p h}= & \frac{k_{f}}{k_{i}} \frac{1}{4 \pi M}(\bar{b})^{2} e^{\left\langle U^{2}\right\rangle} \sum_{m} e^{-i(\boldsymbol{Q} \cdot \boldsymbol{m})} \sum_{s} \frac{\left(\boldsymbol{q} \cdot \boldsymbol{e}_{s}\right)^{2}}{\omega_{s}} \\
& \times \int_{-\infty}^{\infty}\left[\left\langle n_{s}\right\rangle e^{i\left(\boldsymbol{Q} \cdot \boldsymbol{m}-\boldsymbol{\omega}_{s} t\right)}+\left\langle n_{s}+1\right\rangle e^{-i\left(\boldsymbol{Q} \cdot \boldsymbol{m}-\boldsymbol{\omega}_{s} t\right)}\right] e^{-i \omega t} d t .
\end{aligned}
$$

The integration respect to $t$ in (2.27) gives

$$
\int_{-\infty}^{\infty} e^{i\left(\omega_{s}-\omega\right) t} d t=2 \pi \delta\left(\omega-\omega_{s}\right) ; \quad \int_{-\infty}^{\infty} e^{-i\left(\omega_{s}+\omega\right) t} d t=2 \pi \delta\left(\omega+\omega_{s}\right)
$$

while the summation over $\mathrm{m}$ in (2.27) gives

$$
\sum_{m} e^{-i(\boldsymbol{Q}-\boldsymbol{q}) \cdot \boldsymbol{m})}=\frac{(2 \pi)^{3}}{v} \sum_{\tau} \delta(\boldsymbol{Q}-\boldsymbol{q}-\tau) ; \sum_{m} e^{-i(\boldsymbol{Q}+\boldsymbol{q}) \cdot \boldsymbol{m})}=\frac{(2 \pi)^{3}}{v} \sum_{\tau} \delta(\boldsymbol{Q}+\boldsymbol{q}-\boldsymbol{\tau}),
$$

for the first and second term in the brackets of (2.27), respectively.

The cross-section (2.27) thus contains the factors $\delta\left(\omega \pm \omega_{s}\right)$ and $\delta(\boldsymbol{Q} \pm \boldsymbol{q}-\boldsymbol{\tau})$ and means, for scattering to occur, that two conditions must be satisfied:

$$
\omega= \pm \omega_{s}, \quad \text { and } \quad \boldsymbol{Q}=\tau \pm \boldsymbol{q} .
$$

For the first term in brackets of (2.27) the energy of the neutron decreases by an amount equal to the energy of a phonon for the s-th normal mode. So the scattering process is one in which the neutron creates a phonon. It is known as phonon emission. For the second term in brackets of (2.27), instead, the neutron annihilates a phonon in the s-th normal mode. The energy of the phonon goes into an increase in the kinetic energy of the neutron. The process is known as phonon absorption. For both the second condition represents the conservation of momentum.

It is straightforward to generalize the expressions in (2.27) to non-Bravais crystals. The cross-section for coherent one phonon emission becomes

$$
\begin{aligned}
\left(\frac{d^{2} \sigma}{d \Omega_{f} d E_{f}}\right)_{c o h+1 p h}= & \frac{k_{f}}{k_{i}} \frac{(2 \pi)^{3}}{2 v} \sum_{\tau} \sum_{s} \frac{1}{\omega_{s}}\left|\sum_{m} \frac{\overline{b_{m}}}{\sqrt{M_{m}}}\left(Q \cdot e_{s, m}\right) e^{i(\boldsymbol{Q} \cdot \boldsymbol{m})} e^{\left\langle U^{2}\right\rangle}\right|^{2} \\
& \times\left\langle n_{s}+1\right\rangle \delta\left(\omega-\omega_{s}\right) \delta(\boldsymbol{Q}-\boldsymbol{q}-\tau),
\end{aligned}
$$

with a similar expression for the absorption cross-section.

In the one-phonon process for a fixed $k_{i}$, scattering angle, and crystal orientation, scattering occurs only for discrete values of $k_{f}$, while in the two-phonon process, selecting an arbitrary $k_{f}$ within a certain range, we can always find combinations of two normal modes whose $\boldsymbol{q}$ and $\omega_{s}$ values satisfy (2.30). So two-phonon scattering (and higher) gives a smoothly varying continuous spectrum that adds to the background. This is fortunate, as it enables us to separate the effects of one- and multiple-phonon scattering. 
Similar results can be obtained for the incoherent scattering cross-section. If we consider again a Bravais crystal and in this case

$$
U=-i \boldsymbol{Q} \cdot \boldsymbol{u}_{0}(0) \text { and } \quad V_{0}=-i \boldsymbol{Q} \cdot \boldsymbol{u}_{0}(t),
$$

so that

$$
S(\boldsymbol{Q}, \omega)_{i n c}=\frac{N}{2 \pi \hbar}\left(\overline{b^{2}}-(\bar{b})^{2}\right) e^{\left\langle U^{2}\right\rangle} \int_{-\infty}^{\infty} e^{\left\langle U V_{0}\right\rangle} e^{-i \omega t} d t .
$$

The incohérent one-phonon cross-section is obtained from (2.33) by replacing $\exp \left\langle U V_{0}\right\rangle$ with $\left\langle U V_{0}\right\rangle$ :

$$
\begin{aligned}
\left(\frac{d^{2} \sigma}{d \Omega_{f} d E_{f}}\right)_{i n c 1 p h} & =\frac{k_{f}}{k_{i}} \frac{1}{2 M}\left(\overline{b^{2}}-(\bar{b})^{2}\right) e^{\left\langle U^{2}\right\rangle} \sum_{s} \frac{\left(\boldsymbol{q} \cdot \boldsymbol{e}_{s}\right)^{2}}{\omega_{s}} \\
& \times\left[\left\langle n_{s}\right\rangle \delta\left(\omega+\omega_{s}\right)+\left\langle n_{s}+1\right\rangle \delta\left(\omega-\omega_{s}\right)\right] .
\end{aligned}
$$

The first term in the brackets corresponds to phonon emission and the second to phonon absorption. Both terms contains only one $\delta$ - function, $\delta\left(\omega \pm \omega_{s}\right)$ so that only the energy condition needs to be satisfied. For incoherent scattering there is no interference condition like (2.27). Therefore, for a given $k_{f}$, and crystal orientation, incoherent one-phonon scattering occurs for a continuous range of $k_{i}$ values so that we get scattering from all normal modes whose $\omega_{s}$ values satisfy only the first term of (2.30). The cross-section therefore depends only on the number of modes that have the correct frequency.

We can write the cross-sections for a process in which $n$ phonons are exchanged between the neutron and the crystal in the form

$$
\left(\frac{d^{2} \sigma}{d \Omega_{f} d E_{f}}\right)_{c o h n-p h}=\sigma_{c o h} F_{c o h n-p h} \text { and }\left(\frac{d^{2} \sigma}{d \Omega_{f} d E_{f}}\right)_{i n c n-p h}=\sigma_{i n c} F_{i n c n-p h}
$$

The incoherent approximation is to assume that for $n \geq 2$

$$
F_{c o h n-p h}=F_{\text {inc } n-p h},
$$

$F_{c o h n-p h}$ is made up of terms from atoms $m$ and $m^{\prime}$ with both $m=m^{\prime \prime}$ and $m \neq m^{\prime}$, while $F_{\text {inc } n-p h}$ is made up only of terms $m=m^{\prime}$. The justification for the incoherent approximation is that for processes involving two or more phonons the cross-sections for both coherent and incoherent scattering are smoothly varying functions of the scattered energy, the incident energy, and the scattering angle. It therefore appears that the interference terms $m \neq m^{\prime \prime}$ in the coherent cross-section cancel each other to a large extent. The usefulness of the approximation is that it is much easier to calculate $F_{i n c n-p h}$ than $F_{\text {cohn-ph. }}$.

\subsection{Inelastic neutron scattering techniques}

One of the most important applications of the coherent one-phonon scattering process is to measure the phonon dispersion relations for a single crystal, that is, to measure the frequency $\omega_{s}$ as a function of wavevector $\boldsymbol{q}$ and polarization index s. If $k_{f}$ and the crystal orientation are kept constant, the values of $k_{i}$ that satisfy the conservation laws for one-phonon scattering define a scattering surface. There is one such surface for each polarization branch.

Although ideally the frequency spectrum of neutrons scattered in a one-phonon process is a set of $\delta$-functions, in practice the measured peaks have finite widths. The broadening arises from several factors. The first is the resolution of the instrument used, as both $k_{i}$ and $k_{f}$ have a finite spread in magnitude and direction. Secondly the crystal has a mosaic spread. The third and most interesting reason is that the interatomic forces in the crystal are not truly harmonics. The analysis of the displacements 
JDN 21

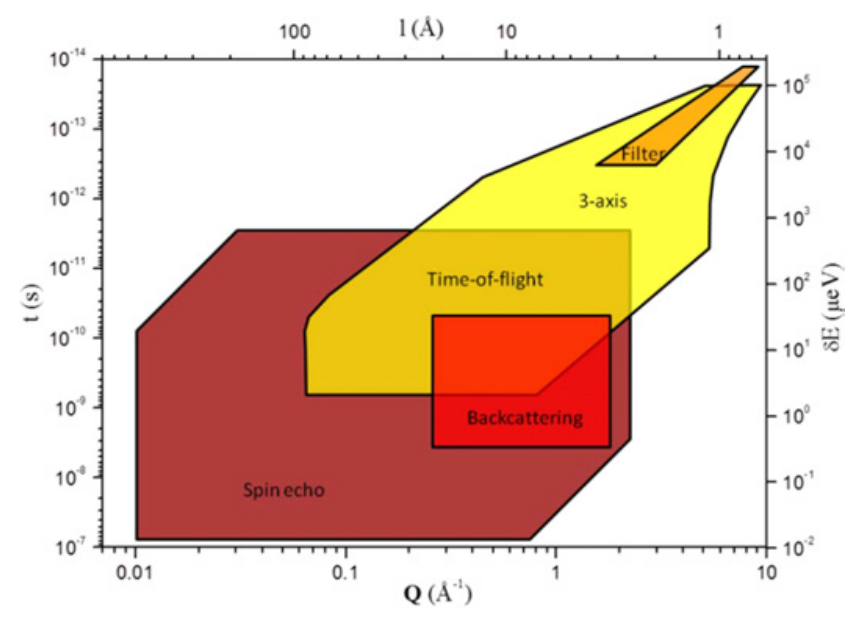

Figure 2. Schematic showing most of the spectrometers used for inelastic neutron scattering studies as a function of the momentum transfer (or wavelength) and exchanged energy (or timescales) they can cover.

of the atoms from their equilibrium positions in terms of a set of non-interacting normal modes is only correct for pure harmonic forces. In fact the forces have an anharmonic component which causes the normal modes to interact with each other. The energy in a mode does not remain constant, but is gradually transferred to other modes. The result is that the one phonon frequency is no longer sharp. It is spread over a finite range and the greater the anharmonic component, the greater the spread. Hence the scattered neutron groups occur, not at a sharp value of $k$, but over a range of values. As the temperature of the crystal is raised, the anharmonic component of the forces increases, and the widths of the neutron peaks increase. By measuring the widths we can study the anharmonic contribution to the forces. In addition to broadening the frequencies of the normal modes, anharmonic forces also produce changes in the mean values of the frequencies.

Various instruments are available for the inelastic neutron scattering studies of materials. Most of them are reported in Fig. 2 as a function of their dynamical range, i.e. the range in exchanged momentum and energy they covers. It is possible to see that the most used spectrometer of type triple axis and timeof flight almost cover the same region of $(\mathrm{Q}, \omega)$ space.

The measurement of phonon dispersion relations from reactors providing a continuous beam of neutrons are usually carried out using a triple-axis spectrometer. Usually, these experiments are carried out keeping either the momentum transfer $\mathbf{Q}$ or the energy transfer $\mathrm{E}_{\mathrm{s}}=\hbar \omega_{\mathrm{s}}$, constant. Such experiments on single crystals aim to probe the scattering from one phonon at a time. In crystalline solids, the momentum transfer $\mathbf{Q}$ is related to the wave vector of the excitation by a reciprocal lattice vector, $\mathbf{Q}=\boldsymbol{\tau} \pm \mathbf{q}$, where $\tau$ is a reciprocal lattice vector and $\mathbf{q}$ is the reduced wave vector in the Brillouin zone. Due to these conservation laws, it is possible to choose specific regions in $\mathbf{Q}-\omega$ space where one can scan for peaks in $\mathbf{S}(\mathbf{Q}, \omega)$. From a large number of such measurements on a single crystal, one can identify several points of the dispersion relations.

The phonon cross sections depend strongly on $\mathbf{Q}$ and the eigenvector $\boldsymbol{e}_{\mathrm{s}}$ of the excitation. For simple structures, the eigenvectors are determined entirely from the symmetry of the space group; the dynamical structure factors are then entirely determined from the crystal structure. For more complex structures, the space group symmetry only classifies the phonons into a number of irreducible representations. The eigenvectors could be any linear combinations of the symmetry vectors associated with the irreducible representation, and their understanding involves extensive lattice dynamics calculations. The lattice dynamical model can then be used to compute the structure factors, and the regions in reciprocal 
space where the neutron scattering cross sections are large may be identified. A variety of methods have been proposed for interpreting the results. Some employ empirical models with force constants treated as adjustable parameters; others are based on more physical models as density functional theory calculations.

Whereas in the case of scattering from single crystals, the scattering vector has a definite orientation with respect to the reciprocal space of the single crystal, in powder samples the reciprocal space axes belonging to the different grains of the powder have different orientations, and ideally all possible orientations are possible with equal probability. Thus, averaging over the various grains is equivalent to averaging over all orientations of the scattering vector. Further averaging over the magnitude of $\mathbf{Q}$ one gets the density of states $S(\omega)$, i.e. the density of excitations at the frequency $\omega$ weighted with the neutron scattering cross sections. The averaging over $\mathbf{Q}$ should be carried out over a suitable chosen range of $\mathbf{Q}$ because the relative height s of the various peaks in $\mathbf{S}(\mathbf{Q}, \omega)$ would be normally $\mathbf{Q}$-dependent. The coherent INS data from a powder sample are usually analyzed in the incoherent approximation. In this approximation, the scattering expression will not contain the phase terms and the directional averaging over $\mathbf{Q}$ gives

$$
|\boldsymbol{Q} \cdot \boldsymbol{e}|^{2}=\frac{1}{3} Q^{2}
$$

We now introduce the partial density of states

$$
F_{k}(\omega)=\frac{1}{3 N_{d}} \sum_{d} \sum_{s}\left|\boldsymbol{e}_{s}(k)\right|^{2} \delta\left(\omega-\omega_{s}\right)
$$

where $N_{d}$ denotes the number of each equivalent group of atoms. The observed neutron-weighted phonon DOS is the sum of the partial components of the density of states weighted by their scattering length and is given by

$$
G(\omega)=\sum_{d} \frac{\sigma_{i n c}(d)}{m_{d}} F_{d}(\omega) .
$$

By using relations (2.37), (2.38) and (2.39) with (2.34) we can finally obtain the incoherent one-phonon scattering from a powder sample in the incoherent approximation as

$$
\left(\frac{d^{2} \sigma}{d \Omega_{f} d E_{f}}\right)_{i n c 1 p h}=\frac{k_{f}}{k_{i}} \frac{N}{8 \pi} Q^{2} e^{\left\langle U^{2}\right\rangle} \frac{G(\omega)}{\omega} \times\left[\left\langle n_{s}\right\rangle \delta\left(\omega+\omega_{s}\right)+\left\langle n_{s}+1\right\rangle \delta\left(\omega-\omega_{s}\right)\right] .
$$

By comparing the experimental phonon spectra with the calculated neutron-weighted density of states obtained from a lattice dynamical model, the dynamical contribution to frequency distribution from various atomic and molecular species can be understood. Any inelastic neutron spectrum contains a contribution from multi phonon scattering that has to be estimated and subtracted from the experimentally observed spectrum $\mathrm{G}(\omega)$ to obtain the one-phonon density of states. Usually, multiphonon scattering contributes as a smoothly varying spectrum and should be removed with adequate methods.

The spectrometers most suited to obtain phonon DOS spectra are the neutron time of flight or a neutron filter ones. These techniques are particularly convenient at pulsed neutron sources. Different spectra are obtained for several different $\mathbf{Q}$, which may differ due to the coherent scattering, and then averaged over $\mathbf{Q}$ to obtain the phonon DOS. Various corrections to the data are needed due to the background scattering, geometrical factors, efficiency of the detectors, second-order reflections from the monochromator and analyzer crystals, etc. The measured spectra also deviate from the true phonon DOS because different atoms contribute differently in proportion to their scattering cross sections divided by their masses. Lattice dynamical models are very useful in providing a microscopic understanding of the complex data and in estimating the multi phonon contributions. 
Application to system well suited for neutron experiment as proton conductor or $\mathrm{H}_{2}$ storage materials have been already target of various review and books that the reader can be addressed to for more information $[4,5]$.

In the following a couple of examples will be presented in which recently inelastic neutron experiment have helped understanding material properties on systems less prone to profit of the neutron characteristic as oxygen ion conductors [6] and thermoelectric materials [7].

\section{Ionic conductors}

In materials science, fast ion conductors are solids in which ions are highly mobile. These materials are important in the area of solid-state ionics and are used primarily in solid oxide fuel cells. As solid electrolytes they allow the movement of ions without the need for a liquid or soft membrane separating the electrodes. The phenomenon, as know today for the high temperature working materials, relies on the hopping of ions through an otherwise rigid crystal structure.

Nevertheless the development of energy efficient devices, such as solid state fuel cells, depends on the availability of materials showing high oxygen ion conduction together with moderate to ambient operating temperatures. The $\mathrm{Nd}_{2} \mathrm{NiO}_{4+\delta}$ system has been proved to be a good candidate as a stable electrode for intermediate temperature solid state fuel cells. More appealing is the fact that $\mathrm{Nd}_{2} \mathrm{NiO}_{4+\delta}$, like a few other non-stoichiometric oxides derived from the perovskite structure, shows oxygen ionic mobility in an electrochemical reaction at room temperature. This unattended behavior raises questions about the real microscopic oxygen mobility mechanisms in these classes of materials when the temperature is as low as ambient one. Indeed oxygen ion is double negative charged and has large ionic radius so that the hopping processes between interatomic sites is hindered by potential barriers at ambient temperature.

A microscopic diffusion mechanism, in which a low-lying phonon mode assists the migration of ions, has been recently developed [8]. This "phonon assisted diffusion" model allowed understanding on an atomic scale how oxygen ion diffusion can be triggered in solid oxides down to ambient temperature. Being a specific low energy lattice mode the trigger for the diffusion process the diffusing ion should displace and diffuse following the polarization of the specific involved phonon and the resulting diffusion path should be therefore strongly anisotropic.

In all these compounds showing ambient temperature oxygen mobility the oxygen ions organize in a series of different patterns within the host lattice as a function of temperature and oxygen content, leading to either complex long-range commensurate or incommensurate structures or short-range disordered arrangements. The $\mathrm{Nd}_{2} \mathrm{NiO}_{4+\delta}$ that, similar to other $\mathrm{K}_{2} \mathrm{NiF}_{4}$-type oxides, shows a complex phase diagram at room temperature as a function of the oxygen content $\delta$. Stoichiometric $\mathrm{Nd}_{2} \mathrm{NiO}_{4.0}$ adopts at ambient the orthorhombic (LTO) phase while for $\mathrm{Nd}_{2} \mathrm{NiO}_{4.1}$ the structure is tetragonal and finally, for fully oxidized $\mathrm{Nd}_{2} \mathrm{NiO}_{4.25}$ the symmetry turns out to be orthorhombic.

The fundamental understanding of the origin of oxygen ion diffusion mechanisms at elevated temperature has been generally explained so far by employing classical atomistic modeling approaches. If these simplified approaches work well for the range of temperatures where stochastic hopping mobility is the fundamental process, it fails in depicting the more complicated low temperature scenario, where the strong interplay between the structure and the vibrational behavior is believed to be at the origin of the mobility mechanism. The complex oxygen ordering, together with the expected anisotropic displacement behavior, demand more sophisticated theoretical approaches like, for example, DFT based calculations.

To study the lattice dynamics present in the regular host system $(\delta=0)$ and the effect of oxygen excess $(\delta=0.10,0.25)$, time-of-flight inelastic neutron scattering experiments on powder polycrystalline samples has been performed. Figure $3 \mathrm{a}$ shows a comparison of the experimental generalized DOS extracted from the inelastic neutron spectra for the three different oxygen contents at 

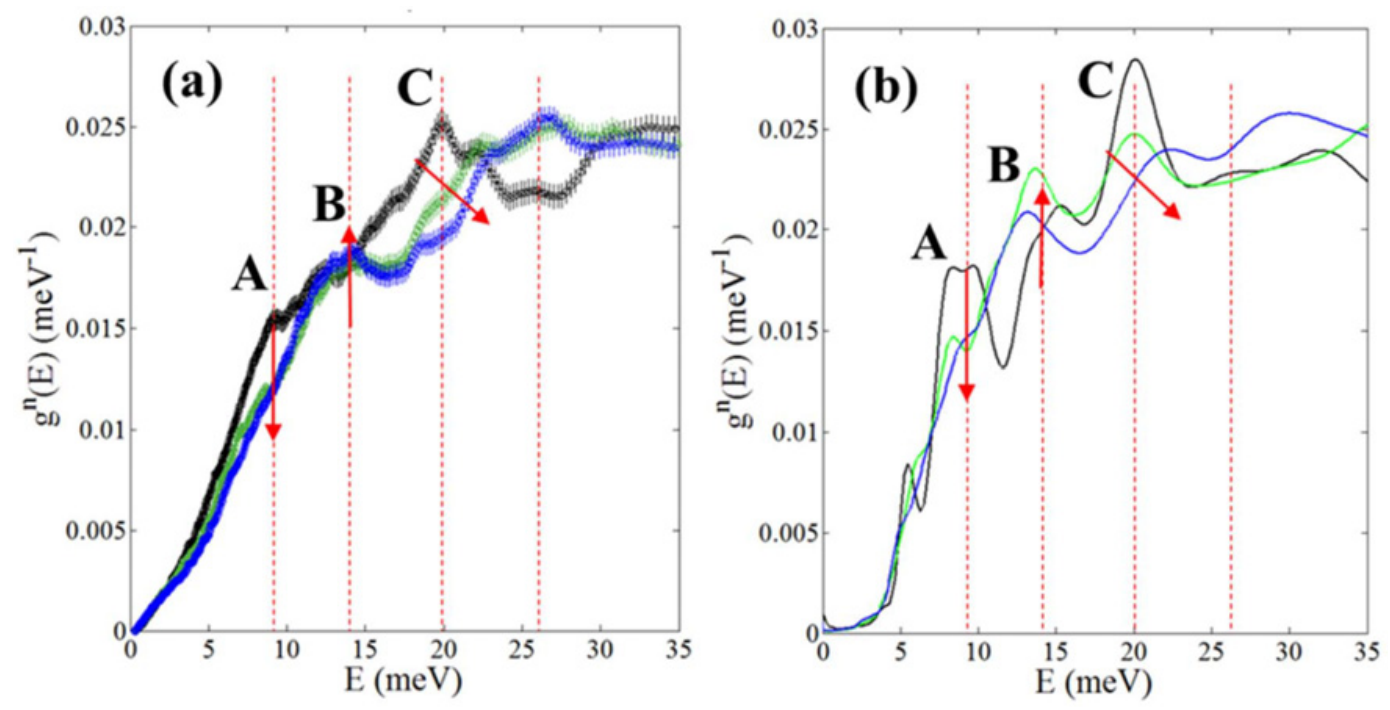

Figure 3. (a) Generalized phonon density of states collected on the IN6 spectrometer of $\mathrm{Nd}_{2} \mathrm{NiO}_{4.0}$ (black), $\mathrm{Nd}_{2} \mathrm{NiO}_{4.10}$ (green), and $\mathrm{Nd}_{2} \mathrm{NiO}_{4.25}$ (blue) at $310 \mathrm{~K}$. (b) Vibrational density of states calculated from DFT molecular dynamics; same color legend as (a). As a guide for the eyes, the increase, decrease, and shift of the main features are marked with red arrows. Image adapted with permission from ref. [6], copyright (2015) American Chemical Society.

$\mathrm{T}=310 \mathrm{~K}$. Three energy ranges of interest in the stoichiometric $\mathrm{Nd}_{2} \mathrm{NiO}_{4.0}$ sample can be distinguished (black dots in Fig. 3a): a region from 5 to $11 \mathrm{meV}$ with a shoulder component (feature A), a region from 11 to $16 \mathrm{meV}$ with a large bump (feature B), and a region from 16 to $21 \mathrm{meV}$ with an intense peak (feature C).

On $\mathrm{Nd}_{2} \mathrm{NiO}_{4.25}$ (from black to blue dots in Fig. 3a), the component A clearly decreases in intensity, while the feature $\mathrm{C}$ shifts toward higher energies and loses intensity. As the feature $\mathrm{B}$ does not change in intensity while components $\mathrm{A}$ and $\mathrm{C}$ decrease, the overall effect is a slight, relative increase of the amount of vibrational states in this region $\mathrm{B}$. As expected, the case of $\mathrm{Nd}_{2} \mathrm{NiO}_{4.1}$ (green dots in Fig. 2a) is intermediate to the other two in both intensity variation and energy shifts, resembling more to the $\mathrm{Nd}_{2} \mathrm{NiO}_{4.25}$ case.

DFT-MD calculations have been performed at room temperature for the three different oxygen contents and the calculated vibrational DOS are shown in Fig. 3b. The main trends observed in experimental spectra from $\mathrm{Nd}_{2} \mathrm{NiO}_{4.0}$ to $\mathrm{Nd}_{2} \mathrm{NiO}_{4.25}$ are reproduced, even though the match with experiment is not perfect due to different limitations: (i) the limited number of atoms that can be simulated by a DFT method ( $<$ few hundreds of atoms), resulting in a simplified structural model; (ii) the intrinsic limitation of the pure DFT method concerning electron-electron correlations. In particular the level of agreement with experiment is lower for $\mathrm{Nd}_{2} \mathrm{NiO}_{4.10}$ due to the fact that we used as a model only one of the four possible configurations of how excess oxygen can be included, the one with the lowest ground state energy after optimization.

To attribute phonon frequencies to specific spectral regions, additional calculations to obtain normal modes by the direct method have been performed. The mode with lowest energy at the Y point of the Brillouin zone has polarization in the [100] direction. The feature A represents thus states with displacements mainly developing along the [100] direction, while feature B concerns the ones along [110]. Feature $\mathrm{C}$ is instead related to the octahedral unit modes and in particular to the stretching along the c-axis direction. 

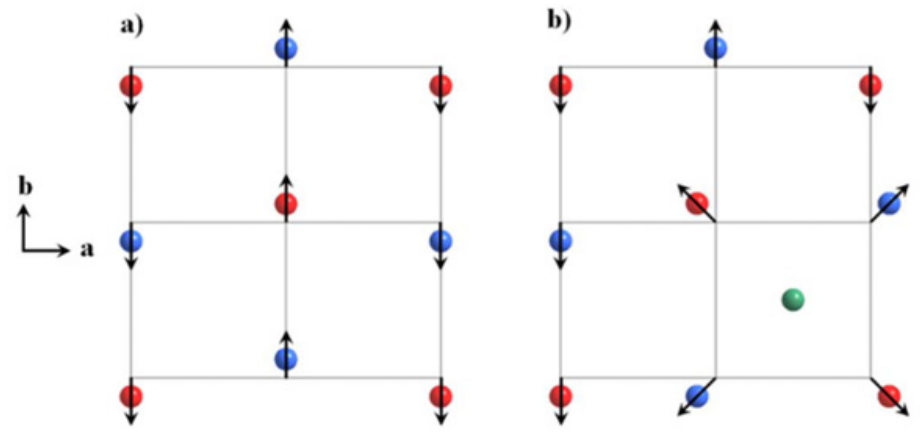

c)

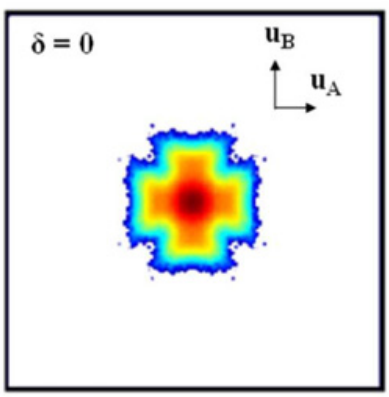

$\delta=0.125$

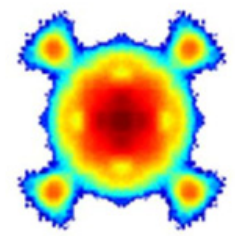

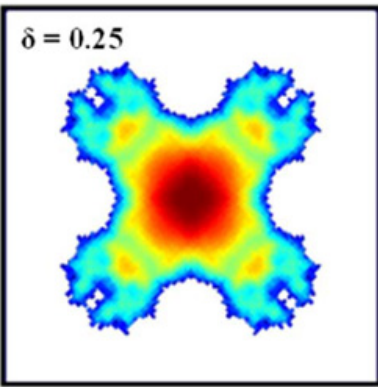

Figure 4. Scheme of the tilt pattern in the Bmeb space group of (a) stoichiometric $\mathrm{Nd}_{2} \mathrm{NiO}_{4.0}$ and (b) $\mathrm{Nd}_{2} \mathrm{NiO}_{4+\delta}$. Apical oxygen atoms in the plane below the interstitial site are represented in red and in the plan above in blue. Excess oxygen in the interstitial site is represented in green. The first shell of apical oxygen atoms surrounding the interstitial is deformed from [100] to [110]. (c) Positional recurrence maps: displacement pattern of apical oxygen atoms in the $\mathrm{Nd}_{2} \mathrm{O}_{2}$ rock-salt layer from an initial center-of-mass positions projected on unitary vectors (a,b), with respect to the conventional F-cell. PRMs are calculated from ab initio molecular dynamics at $310 \mathrm{~K}$ for $\mathrm{Nd}_{2} \mathrm{NiO}_{4.0}$, $\mathrm{Nd}_{2} \mathrm{NiO}_{4.10}$, and $\mathrm{Nd}_{2} \mathrm{NiO}_{4.25}$. Image adapted with permission from ref. [6], copyright (2015) American Chemical Society.

The observed variations in function of excess oxygen in Fig. 3 reflect a decrease of spectral weight for vibrational modes with displacement pattern along [100] and a concomitant increase for modes with displacement pattern along [110]. Moreover, the introduction of interstitial oxygen constrains the octahedral stretching mode which consequently hardens and dampens (feature C). More precisely, as $\delta$ increases, the oxidation state of $\mathrm{Ni}$ move from $\mathrm{Ni}^{2+}$ toward $\mathrm{Ni}^{3+}$ and consequently shortens the $\mathrm{Ni}-\mathrm{O}_{\text {ap }}$ bond length, while the excess oxygen atoms force an elongation of the stackingaxis, thus stretching the rock-salt layer where oxygen is diffusing. The modification of the tilt pattern from [100] to [110] favors the displacement of the apical oxygen atom to the rock-salt layer. These two effects relate the observed damping of the modes $\mathrm{A}$ and $\mathrm{C}$ to enhanced oxygen mobility.

In the stoichiometric $\mathrm{Nd}_{2} \mathrm{NiO}_{4.0}$ case, the apical oxygen position recurrence map (for explanations see caption of Fig. 4) presents a cross shape with preferred displacements in the [100] direction as shown in Fig. 4c. This is expected since the LTO structure, without the deformations imposed by interstitial oxygen atoms (see Fig. 4a), must have the tilt pattern of apical oxygen along [100]. For the $\mathrm{Nd}_{2} \mathrm{NiO}_{4.25}$ case, the second neighbor interaction disappears, and the [110] pathway is broadened. For the intermediate $\mathrm{Nd}_{2} \mathrm{NiO}_{4.10}$ case, the positional recurrence pattern shows both [100] and [110] delocalization. This is consistent with the abovementioned quarter filling of the interstitial sites for $\delta=0.1$, which means that $75 \%$ of apical oxygen atoms have unperturbed dynamics and contribute to 
[100] delocalization and that $25 \%$ of them experience the [110] delocalization. It is worth noticing that the small satellites in the outer shell come from diffusion events of oxygen atoms from the apical to the interstitial site.

The modification of lattice dynamics with the inclusion of extra oxygen directly influence the mechanism of oxygen ion diffusion involving both apical and interstitial oxygen atoms and that can be schematized as follows: (i) one of the apical oxygen atoms closest to the excess oxygen diffuses away to a vacant interstitial site; (ii) then the former interstitial oxygen diffuses to the newly vacant apical site. Such diffusion is confined in the rock-salt layer with jump only along [110]. We note that in the absence of excess oxygen in the interstitial site of a rock-salt layer, e.g., for $\mathrm{Nd}_{2} \mathrm{NiO}_{4.0}$, no diffusion events occur in this layer.

\section{Thermoelectric materials}

The thermoelectric effect refers to phenomena by which either a temperature difference creates an electric potential or an electric potential creates a temperature difference. Both thermal conductivity and electrical conductivity compete in giving the thermoelectric efficiency and as consequence phonons, which are responsible for thermal conductivity, are the main object of study in this field.

New thermoelectric materials often have large unit cells and, therefore, calculations of the thermal conductivity $\kappa$ using acoustic only phonon branches within the usual Debye framework are inadequate. It is necessary instead to take account of optical modes and, as consequence, detailed knowledge of the phonon dispersion is required. The possibility to measure phonon dispersions in detail using both inelastic X-ray scattering (IXS) and inelastic neutron scattering (INS) provides unprecedented opportunities to understand thermal transport mechanism in these systems. The experiments on samples with a complicated unit cell require a model calculation behind to correctly assign spectral features.

The efficiency of operation of a thermoelectric material is usually measured by the figure-of-merit,

$$
Z T=\frac{\sigma T S^{2}}{\kappa}
$$

a dimensionless number proportional to the square of the Seebeck coefficient $S$ and the ratio $\sigma / \kappa$ of the electrical to thermal conductivities. A phonon-glass electron-crystal model has been proposed [9], where the rattling of loosely bound atoms in the structural cages is believed to disrupt the vibrational modes of the cages in a way that heat conduction become comparable to glasses. Nevertheless theoretical studies have called into question the validity of the phonon-glass electron-crystal and rattler concepts as INS measurements demonstrate that phonon excitations are sharp for many of these materials [10].

Particularly good thermoelectric properties have been observed for sodium cobaltate. The thermoelectric $\mathrm{Na}_{\mathrm{x}} \mathrm{CoO}_{2}$ contains multi-vacancy clusters that order to form long ranged superstructures Theoretical studies have considered the electronic contributions to ZT using density functional theory (DFT) and Boltzmann transport theory, explaining the suppression of $\mathrm{S}$ in large magnetic fields. Anyhow those calculations omitted the low-energy rattling modes caused by the multi-vacancy clusters. Recent calculations including the multi-vacancy of the phonon dispersion are able to unambiguously identify an Einstein-like rattler mode at low energy and to quantitatively account for the suppression of $\kappa$ for this class of materials. These calculations well compare with measurements of momentum-resolved IXS and INS spectra from single-crystal $\mathrm{Na}_{0.8} \mathrm{CoO}_{2}$ with a well-defined superstructure.

Scans of variable energy transfer were performed at a number of settings of $Q$ along the highsymmetry directions $\Gamma$-K-M from (111) and for $\Gamma$-M from (100). The calculated scattering intensity from the vacancy cluster for the square superstructure $\mathrm{Na}_{0.8} \mathrm{CoO}_{2}$ well agrees with the ab initio calculations. The mode at $\mathrm{E} \approx 13 \mathrm{meV}$ is captured by the model. In contrast, the ideal vacancy-free compound has no modes below $\mathrm{E} \approx 20 \mathrm{meV}$ away from the zone centre. 

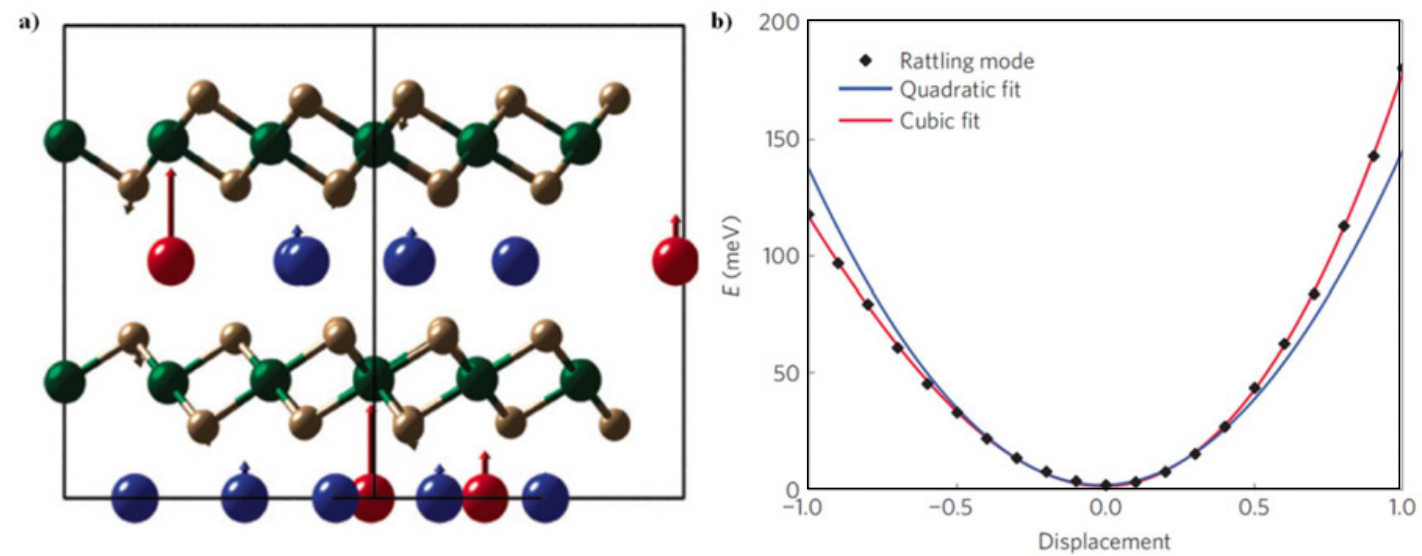

Figure 5. (a) The supercell of $\mathrm{Na}_{0.8} 0 \mathrm{CoO}_{2}$ showing the rattling mode at $E \approx 13 \mathrm{meV}$ comprising mainly displacements of the (red) $\mathrm{Na} 2 b$ ions inside tri-vacancy clusters. $\mathrm{Na} 2 d$ ions are blue, Co is green and $\mathrm{O}$ is gold. The length of the arrow indicates the relative amplitude for each ion, not an absolute displacement. (b) Frozen phonon calculation for the rattling mode (black). The displacement is scaled to normal mode amplitude, which corresponds to a maximum in-plane displacement of the $\mathrm{Na} 2 b$ site of $0.3 \AA$. The asymmetry of the potential energy as a function of displacement of the rattling ions clearly demonstrates the anharmonicity of this mode. Image adapted with permission from ref. [7], copyright (2013) Nature publishing group.

It is possible to trace the mode at $\mathrm{E} \approx 13 \mathrm{meV}$ back to the zone centre, and look the relative amplitudes of the ionic displacements (see Fig. 5a). The blue spheres in Fig. 5a are the Na 2d sites, and these are fully occupied in the stoichiometric compound. The red spheres are on the non-equivalent $\mathrm{Na}$ $2 \mathrm{~b}$ sites and, because they are associated with clusters of three vacancies, they are expected to be more loosely bound. The atomic displacement pattern is characterized by predominant in-plane amplitude of vibration of the $\mathrm{Na} 2 \mathrm{~b}$ site with only very minor or negligible contribution from other sites. Thus, we find that the $\mathrm{Na} 2 \mathrm{~b}$ sites inside tri-vacancy clusters sandwiched between $\mathrm{CoO}_{2}$ layers are rattler sites, and the mode at $\mathrm{E} \approx 13 \mathrm{meV}$ is a rattling mode.

The atomic displacement parameters for $\mathrm{Na}$ are larger for $\mathrm{Na}_{0.8} \mathrm{CoO}_{2}$ than for $\mathrm{NaCoO}_{2}$, confirming the larger amplitude of vibration in the superstructure. Frozen phonon calculations were performed for the rattler mode, and the potential energy is plotted as a function of displacement in Fig. 5b. The crystallographic asymmetry of the $\mathrm{Na} 2 \mathrm{~b}$ site gives rise to a cubic term in energy and consequently a significant degree of anharmonicity. The energy of this mode is $\mathrm{E} \approx 13 \mathrm{meV}$ and, therefore, anharmonicity is expected to become important above $\mathrm{T} \approx 150 \mathrm{~K}$.

Measurements of $\kappa$ using a Quantum Design physical property measurement system on a crystal of the same composition are presented in Fig. 6a. The magnitude of $\kappa$, in the region of a few watts per Kelvin-meter, is similar to the values reported for crystals of comparable composition and the temperature dependence clearly indicates a contribution from phonon scattering.

Phonon line-widths at high resolution have been measured in a region of the dispersion dominated by a single optical phonon at $\mathrm{Q}(1.01 ; 1.01 ; 1)$ and $\mathrm{E} \approx 8 \mathrm{meV}$ using the triple-axis neutron spectrometer IN8 at ILL with a $30^{\prime}-20^{\prime}-30^{\prime}-30^{\prime}$ collimation configuration. The energy line shape is resolution-limited below $\mathrm{T} \approx 80 \mathrm{~K}$, while above this temperature we fitted the data using a Lorentzian convolved with the instrumental resolution function.

Figure $6 \mathrm{~b}$ shows a typical energy scan and the phonon lifetimes $\tau$, related to the energy linewidths, $\Gamma^{\mathrm{ph}}$ by the relation $\tau \sim h / \Gamma^{\mathrm{ph}}$, as a function of temperature. These data are also consistent with measurements using IXS averaged over several modes at $\mathrm{T} \approx 200 \mathrm{~K}$. There is no evidence for the marked energy broadening expected for a phonon glass. The lattice contribution to $\kappa$ 

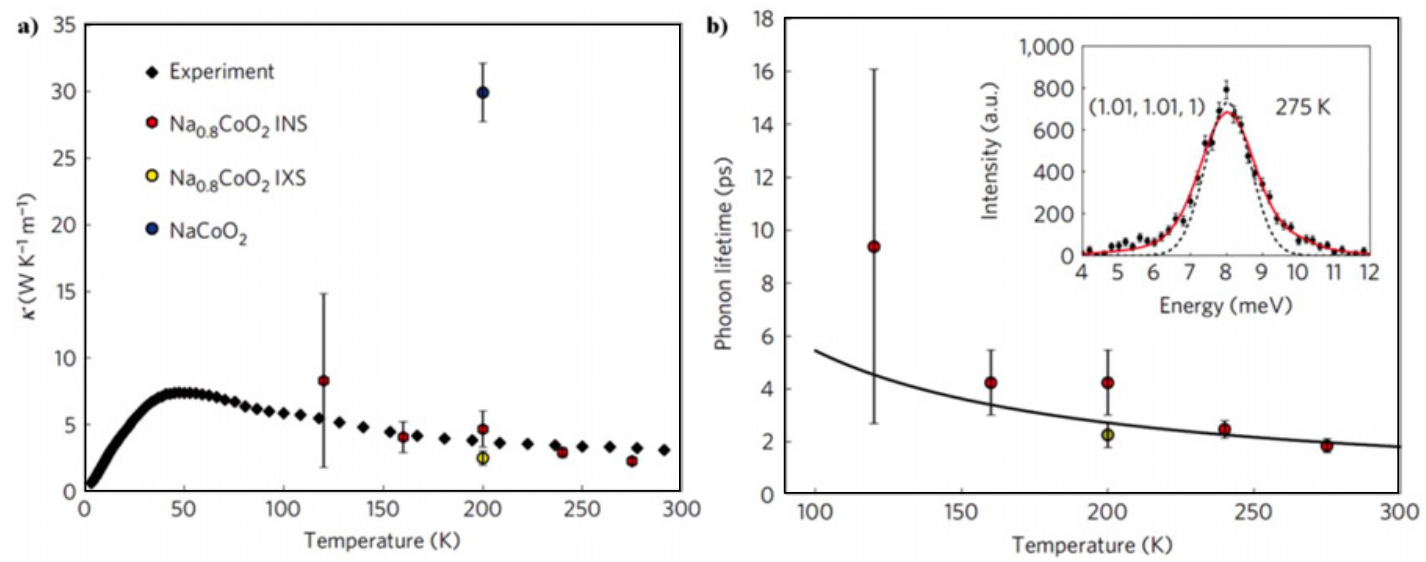

Figure 6. (a) The measured thermal conductivity of $\mathrm{Na}_{0.8} \mathrm{CoO}_{2}$ (black) is compared with the calculated lattice contribution to the thermal conductivity for the $\mathrm{Na} 0: 8 \mathrm{CoO} 2$ superstructure comprising a square array of tri-vacancy clusters using the phonon lifetimes derived using neutrons (red) and X-rays (yellow). The calculated values for $\mathrm{NaCoO}_{2}$ (blue) are a factor of six higher. (b) The phonon lifetimes determined from the widths of strong single modes. The lifetimes obtained using INS (red) are consistent with the measurement using IXS (yellow). The solid black line shows the fitted inverse temperature dependence. A typical INS scan at fixed Q $(1.01 ; 1.01 ; 1)$ at $T \approx 275 \mathrm{~K}$ is shown in the inset. The red line shows the best fit of a Lorentzian convolved with the resolution Gaussian, and the dashed black line shows the best fit of the resolution Gaussian. Error bars in (a) and (b) are determined by the fits to phonon lifetimes, and in the inset by Poisson statistics. Image adapted with permission from ref. [7], copyright (2013) Nature publishing group.

was estimated using the expression

$$
\kappa^{L}=\sum_{j, Q} v_{j x}(Q) \tau_{j}(Q, T) c_{j}(Q, T)
$$

where $v_{\mathrm{jx}}$ is the group velocity along the thermal gradient, $\mathrm{c}_{\mathrm{j}}$ is the heat capacity of the $\mathrm{j}$-th phonon mode, and $\tau_{\mathrm{j}}$ is the phonon lifetime. The values for $v_{\mathrm{jx}}$ and $\mathrm{c}_{\mathrm{j}}$ have been determined using the calculated dispersions assuming Bose statistics, and the average value of $\tau$ at each temperature was obtained from Fig. 6b. The estimated value of $\kappa^{\mathrm{L}}$, calculated using our model for the square superstructure, agrees with the data within experimental uncertainty. The electronic contribution to $\kappa$, estimated using the Wiedemann-Franz law from the electrical conductivity, as found to be an order of magnitude lower.

For comparison, the $\kappa^{\mathrm{L}}$ at $\mathrm{T} \approx 200 \mathrm{~K}$ for the stoichiometric compound has been estimated, artificially assuming that $\tau$ is the same (blue circle in Fig. 6a). The fact that this value is roughly six times higher clearly indicates that rattling modes are important in lowering $\kappa$, because they contribute significantly to the specific heat but have small propagation velocities.

\section{References}

[1] S.W. Lovesey, Theory of neutron scattering from condensed matter, Oxford university press, Oxford (1984).

[2] G.L. Squires, Introduction to the Theory of Thermal Neutron Scattering, Cambridge University Press, Cambridge (1978).

[3] B. Dorner, Coherent inelastic neutron scattering in lattice dynamics, Springer Verlag, Berlin (1982). 
[4] P.C.H. Mitchell, S.F. Parker, A.J. Ramirez-Cuesta and J. Tomkinson, Vibrational spectroscopy with neutrons, World Scientific Publishing, Singapore (2005).

[5] K. Gordon and V. Peterson Eds., Neutron Applications in Materials for Energy, Springer Verlag, Berlin (2015).

[6] A. Perrichon, A. Piovano, M. Boehm, M. Zbiri, M. Johnson, H. Schober, M. Cerettiand W. Paulus, J. Phys. Chem. C 119, 1557 (2015).

[7] D. J. Voneshen, K. Refson, E. Borissenko, M. Krisch, A. Bosak, A. Piovano, E. Cemal, M. Enderle, M. J. Gutmann, M. Hoesch, M. Roger, L. Gannon, A. T. Boothroyd, S. Uthayakumar, D. G. Porter, J. P. Goff, Nature Materials, 12, 1027 (2013).

[8] W. Paulus, H. Schober, S. Eibl, M. Johnson, T. Berthier, O. Hernandez, M. Ceretti, M. Plazanet, K. Conder, and C. Lamberti, J. Am. Chem. Soc. 130, 16080 (2008).

[9] G.A. Slack, CRC Handbook of Thermoelectrics (ed. Rowe, D. M.) 407, CRC (1995).

[10] J. Dong, O.F. Sankey, and C.W. Myles, Phys. Rev. Lett. 86, 2361 (2001). 